\title{
Ultrasound features predicting the 3-week outcome of Pavlik harness treatment for developmental hip dysplasia
}

\author{
Guang-Wei Xu, Yan-Cai Yang, Zhen-Hua Xu, Yun-Long Liu \\ Department of Pediatric Orthopedics, Ningbo Women \& Children's Hospital, Ningbo 315012, China \\ Contributions: (I) Conception and design: GW Xu, YC Yang; (II) Administrative support: GW Xu, YL Liu; (III) Provision of study materials or \\ patients: YC Yang, ZH Xu; (IV) Collection and assembly of data: GW Xu, ZH Xu; (V) Data analysis and interpretation: YC Yang, YL Liu; (VI) \\ Manuscript writing: All authors; (VII) Final approval of manuscript: All authors. \\ Correspondence to: Yan-Cai Yang. Master's Degree, Deputy Chief Physician, Department of Pediatric Orthopedics, Ningbo Women \& Children's \\ Hospital, No. 339 Liuting Street, Haishu District, Ningbo 315012, China. Email: yangyancai2008@163.com.
}

Background: The treatment protocol for children with developmental dysplasia of the hip (DDH) is routinely adjusted by assessing the hip reduction after 3 weeks of Pavlik harness treatment. However, there is a high risk of failure and complications in the treatment adjustment. The aim of this study was to explore the value of ultrasound features in predicting the treatment outcome of Pavlik harness after 3 weeks in DDH children.

Methods: A total of 215 DDH children were recruited and the demographics and the changes of ultrasound features [ $\alpha$ and $\beta$ angle and femoral head coverage (FHC)] during the Pavlik harness treatment were recorded. The children were divided into the success group and the failure group according to the reduction outcome after 3 weeks. Univariate and multivariate analyses were performed to analyze the independent predictors for the treatment outcome. Repeated-measures analysis of variance was used to compare the changes of ultrasound features between the two groups during the treatment. Receiver operating characteristic (ROC) curves were plotted to analyze the predictive accuracy of the ultrasound features.

Results: Graf type III $(\mathrm{P}=0.036)$, bilateral dislocation $(\mathrm{P}=0.031)$, and age at diagnosis $(\mathrm{P}=0.021)$ were associated with an increased risk of Pavlik harness failure in the multivariate analysis. The changes in $\alpha$ and $\beta$ angle and FHC were generally greater in the success group than in the failure group. The $\alpha$ angle and FHC were larger in the success group, while the $\beta$ angle was larger in the failure group $(\mathrm{P}<0.05)$. Each ultrasound parameter ( $\alpha$ and $\beta$ angle and FHC) alone could not accurately predict the treatment outcome within 3 weeks. However, the combined ultrasonic features at the second week could accurately predict the outcome of Pavlik harness treatment after the third week. The combination of the ultrasound features at the first week and the influencing factors (Graf classification, age at diagnosis, and side of pathology) could accurately predict the outcome at the first week [area under curve $(A U C)=0.931$, sensitivity $=82.14 \%$, specificity $=97.86 \%$ ].

Conclusions: The combined model of ultrasonic features at the second week could accurately predict the reduction outcome of Pavlik harness after the third week. The combined model including independent predictors and ultrasonic features could accurately predict the reduction outcome at the first week.

Keywords: Developmental dysplasia of the hip (DDH); Pavlik harness; ultrasound; reduction outcome; combined model

Submitted Mar 10, 2020. Accepted for publication Apr 28, 2020.

doi: $10.21037 / \mathrm{apm}-20-889$

View this article at: http://dx.doi.org/10.21037/apm-20-889 


\section{Introduction}

Developmental dysplasia of the hip (DDH) is a common bone and joint deformity in infants. Early diagnosis and treatment can effectively prevent claudication, femoral head necrosis, and even limb disability (1-3). Those who are positive for physical examination or have high risk factors for DDH (breech birth, positive family history, etc.) will receive ultrasonic scanning and be classified by Graf as soon as possible (4-6). Currently, Pavlik harness is the routine treatment for DDH children at 0-6 months $(7,8)$. The success rate of reduction is $63-93 \%$ and depends on multiple factors such as disease severity and the age at diagnosis (9). The study by Ömeroğlu et al. (10) proposed that the success rate of Pavlik harness decreased gradually with the increase of age or Graf classification. Therefore, in clinical settings, Pavlik harness treatment is the first choice for Graf type IIc-III but not for Graf type IV (11).

In clinic, adjustment of the treatment protocol by assessing the hip reduction after 3 weeks of Pavlik Harness treatment is common (most DDH children achieve reduction within 3 weeks) (12). However, the adjusted treatment may prolong the treatment course, reduce the success rate of traction plaster and increase the risk of femoral head necrosis (13). Tiruveedhula et al. (14) found that if the treatment of DDH children is adjusted to traction plaster after the failure of Pavlik harness treatment, the femoral head necrosis rate is $28 \%$, which is much higher than that of patients treated with traction plaster directly $(8 \%)$. This indicates that there is a high risk of failure and complications in the treatment adjustment. Furthermore, continuous compression of the posterior wall of the acetabulum will further increase the difficulty of reduction if DDH children with no or unstable reduction for more than three weeks. It will decrease the success rate of eventual reduction and increase the incidence of femoral head necrosis $(15,16)$. Therefore, it is of great importance to predict the reduction outcome as soon as possible within the 3 weeks and adjust the treatment protocol in time to improve the success rate of eventual reduction. However, the research of early prediction of the reduction outcome is limited.

In the present study, the DDH children received clinical and ultrasonic examinations once a week (3 weeks total) during the Pavlik harness treatment. The changes of the hip joint $\alpha$ angle, $\beta$ angle, and femoral head coverage (FHC) at the first, second, and third week were observed to evaluate the accuracy of ultrasound features in predicting the reduction outcome of Pavlik harness treatment within 3 weeks. It is hoped that the findings of this study can contribute to improving the treatment protocol by reducing the rate of eventual failure. We present the following article in accordance with the STARD reporting checklist (available at http://dx.doi.org/10.21037/apm-20-889).

\section{Methods}

\section{Research participants}

This is a prospectively study, and 215 DDH children treated in the Department of Pediatric Orthopedics, Ningbo Women \& Children's Hospital from June, 2016, to September, 2019 were consecutively recruited. The inclusion criteria were as follows: (I) age at diagnosis $\leq 6$ months; (II) Graf type IIc, D, and III; and (III) complete and determinate clinical and imaging data. DDH children with obvious muscle imbalance, pathological ligament relaxation, or joint stiffness were excluded and. Patient demographics, including gender, age at diagnosis, and side of pathology data, were recorded after recruitment. All children's families signed informed consent, and this study was approved by the ethics committee of Ningbo Women \& Children's Hospita (No. NWCH20160217).

\section{Research methods}

\section{Ultrasound scanning}

The Philips EPIQ7 ultrasound system (Philips Healthcare, Seattle, WA, USA) with an L12-5 linear array probe (5$12 \mathrm{MHz}$ ) was used. The infant was placed into the lateral position, and the hip joint was slightly flexed. The probe was placed on the outside of the hip joint, and the long axis of the probe was positioned parallel to the body axis. The scanning was initiated to obtain the standard coronal section acoustic image of the hip joint. The $\alpha$ and $\beta$ angles of children were measured, and the DDH children were typed blindly according to Graf classification (17) (Type IIc: $43^{\circ} \leq \alpha \leq 50^{\circ}, \beta \leq 77^{\circ}$; Type D: $43^{\circ} \leq \alpha \leq 50^{\circ}, \beta>77^{\circ}$; Type III: $\alpha<43^{\circ}, \beta$ not performed due to poor development of the bony acetabular roof) (Figure 1). FHC (18) was calculated as $\mathrm{d} / \mathrm{D}$, in which $\mathrm{d}$ represents the distance from the inner edge of the femoral head to the baseline of the ultrasonic image, and $\mathrm{D}$ represents the femoral head diameter (Figure 1).

\section{Treatment methods}

The initial treatment protocol for all DDH children was 


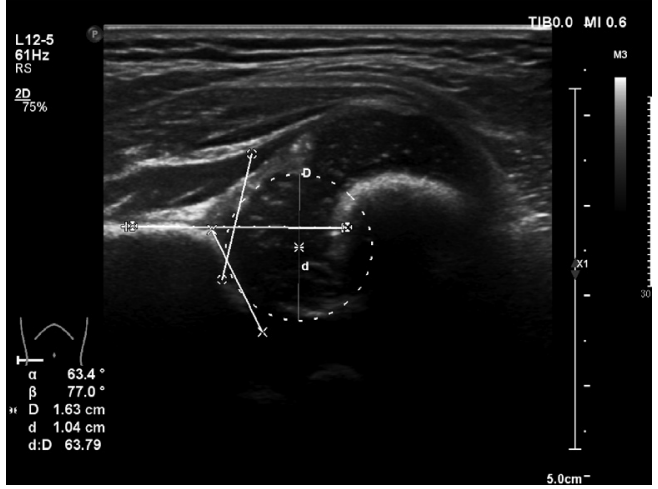

Figure 1 Ultrasound characteristics and features of DDH children with DDH. d/D in the figure represents FHC. DDH, developmental dysplasia of the hip; FHC, femoral head coverage.

with the Pavlik harness. We laid the brace on the bed and placed the child in the brace. The child's arms were put into the shoulder straps, and the lower limbs were placed into the foot straps. Then, the flexion bands and abduction bands were tightened to flex the child's hip joint to $80^{\circ}$, extend it $60^{\circ}$, and flex the knee to $50^{\circ}$. The sling was worn for $23 \mathrm{~h}$ each day, and a hip ultrasound review was performed once a week within 3 weeks. The hip reduction was evaluated, and the $\alpha$ and $\beta$ angles, along with the FHC, were recorded each week.

\section{Grouping criteria}

The hip joint stability of DDH children was evaluated by Harcke's test after the third week of Pavlik harness treatment (19). According to the reduction outcome, the DDH children with stable reduction evaluated by ultrasound clinical examination were included in the success group. They continued to receive Pavlik harness treatment for 3 months, and then fixed with a nighttime brace until the acetabulum developed normally. The DDH children with no or unstable reduction were included in the failure group, and other treatment methods (e.g., traction plaster) were required for them.

\section{Statistical analysis}

All information was recorded anonymously and processed using Statistical Product and Service Solutions (Chicago, IL,USA) software (version 22.0) and plotted by R package version 3.6.2 and MedCalc version 12 (MedCalc Software, Ostend, Belgium). The numerical data were expressed as mean \pm SD and independent sample $t$-tests were used for comparison. The categorical variables were expressed as numbers, and the comparison between the two groups was conducted with the chi-square test. Univariate analysis was used to compare the success group and failure group for the purpose of assessing the differences regarding age at diagnosis, gender, Graf classification, side of pathology, and ultrasound features (i.e., $\alpha$ and $\beta$ angle and FHC). Independent multivariate predictors of outcome were identified using logistic regression in which all variables with $\mathrm{P}<0.05$ from the univariate analysis were entered into the stepwise model. Repeated-measures analysis of variance was used to calculate the differences in hip joint ultrasound trends between the success and failure groups. Receiveroperating characteristic (ROC) curve was plotted to analyze the predictive accuracy of the efficacy of Pavlik Harness treatment at the first, second, and third week.

\section{Results}

\section{Demographics and ultrasound features}

A total of 215 DDH children were included in this study, 192 of whom were female and 23 of whom were male (187 unilateral and 28 bilateral). The average age at diagnosis was $65.1 \pm 19.7$ days. In the Graf classification, 62 children were type IIc, 58 were type D, and 95 were type III. After 3 weeks of Pavlik Harness treatment, 187 children were included in the success group, and 28 children with no or unstable reduction were included in the failure group. The comparison of demographics and ultrasonic characteristics in the two groups is shown in Table 1. The Graf classification in the success group and the failure group was different, and the proportion of type III in the failure group was larger $(\mathrm{P}<0.05)$. The average age at diagnosis and the proportion of bilateral hip in the in the failure group were greater than those in the success group (both $\mathrm{P}<0.05$ ). For ultrasound features, $\alpha$ angle and FHC were larger in the success group, while $\beta$ angle was larger in the failure group (all $\mathrm{P}<0.05$ ).

\section{Predictors for the 3-week outcome of Pavlik harness treatment}

Logistic regression analysis showed that the gender and ultrasound features were not predictors for the outcome of Pavlik harness treatment $(P>0.05)$, whereas Graf type III $(\mathrm{P}=0.036)$, bilateral dislocation $(\mathrm{P}=0.031)$, and age at diagnosis $(\mathrm{P}=0.021)$ were associated with an increased risk of Pavlik harness failure in the multivariate analysis (Figure 2). 
Table 1 Comparison of the demographics and ultrasound features between the success and failure group

\begin{tabular}{|c|c|c|c|c|}
\hline Variable & Success group $(n=187)$ & Failure group $(\mathrm{n}=28)$ & $\mathrm{t} / \chi^{2}$ value & $P$ value \\
\hline Ilc & 60 & 2 & 8.228 & 0.016 \\
\hline$D$ & 50 & 8 & & \\
\hline III & 77 & 18 & & \\
\hline \multicolumn{5}{|l|}{ Gender } \\
\hline Male & 20 & 3 & 0.000 & 0.998 \\
\hline Female & 167 & 25 & & \\
\hline \multicolumn{5}{|l|}{ Side of pathology } \\
\hline$\alpha$ angle $\left({ }^{\circ}\right)$ & $42.27 \pm 4.43$ & $40.14 \pm 4.29$ & 2.378 & 0.018 \\
\hline$\beta$ angle $\left({ }^{\circ}\right)$ & $73.56 \pm 8.58$ & $79.50 \pm 5.54$ & 2.142 & 0.034 \\
\hline FHC (\%) & $36.17 \pm 9.42$ & $29.63 \pm 7.92$ & 3.496 & 0.001 \\
\hline
\end{tabular}

\section{Trend comparison of ultrasound features during Pavlik barness treatment}

Table 2 shows the trend changes in ultrasound features ( $\alpha$ and $\beta$ angle and FHC) between the success and failure group. It revealed that $\alpha$ angle and FHC generally showed an upward trend and $\beta$ showed a downward trend during the treatment (all $\mathrm{P}_{\text {within group }}<0.05$ ). The changes of $\alpha$ and $\beta$ angle and FHC in the success group were generally larger than those in the failure group (Figure 3). The interactions of the three features were statistically significant $\left(\right.$ all $\mathrm{P}_{\text {interaction }}$ $<0.05)$. The $\alpha$ angle and FHC were larger in the success group, whereas the $\beta$ angle was larger in the failure group (all $\left.\mathrm{P}_{\text {between group }}<0.05\right)$.

\section{Accuracy analysis of ultrasound features ( $\alpha$ and $\beta$ angle and FHC) in predicting the outcome of Pavlik harness within 3 weeks}

The ultrasound features ( $\alpha$ and $\beta$ angle and FHC) could better predict the treatment outcome, showing greater accuracy as time progressed. The area under curves (AUCs) of $\alpha$ and $\beta$ angle and FHC at the third week were greater than those at the first week. The AUCs of $\beta$ angle at different weeks were higher than those of $\alpha$ angle and FHC, but the AUC of $\beta$ was still $\leq 0.9$ even at the third week. This indicates that each ultrasound parameter alone ( $\alpha$ angle, $\beta$ angle, or FHC) could not accurately predict the treatment outcome within 3 weeks (Table 3 and Figure 4).

\section{Combination of the ultrasound features ( $\alpha$ and $\beta$ angle and FHC) and influencing factors in predicting the treatment outcome}

The combination of $\alpha$ and $\beta$ angle and FHC was established to improve the accuracy since the accuracy of $\alpha$ and $\beta$ angle and $\mathrm{FHC}$ at the third week for predicting the treatment outcome was not high. The results showed that the AUC of the combination at the first week was lower (AUC $=0.746)$, whereas that at the second week was higher (AUC $=0.909$ ), with a sensitivity and specificity of $75 \%$ and $93.58 \%$ respectively. This suggests that the combined ultrasonic features ( $\alpha$ and $\beta$ angle and FHC) at the second week could can accurately predict the outcome of Pavlik harness treatment after the third week. In order to predict the outcome earlier, this study combined the ultrasound features ( $\alpha$ and $\beta$ angle and FHC) at the first week with the influencing factors (Graf classification, age at diagnosis, and side of pathology). It was revealed that the combined model 
Odds ratio plot

\begin{tabular}{|c|c|c|c|c|}
\hline Variables & Subgroup & & Odds ratio $(95 \% \mathrm{Cl})$ & $P$ value \\
\hline Age at diagnosis & ק & & $1.028(1.007-1.050)$ & 0.01 \\
\hline \multirow[t]{2}{*}{ Side of pathology } & Unilateral dislocation & & Reference & \\
\hline & Bilateral dislocation & +1 & $1.184(1.032-3.368)$ & 0.031 \\
\hline \multirow[t]{3}{*}{ Graf classific ation } & IIc & & Reference & \\
\hline & D & $\longrightarrow$ & $4.800(0.975-23.639)$ & 0.054 \\
\hline & III & $\longmapsto$ & $7.013(1.566-31.410)$ & 0.011 \\
\hline$\alpha$ & $\longmapsto$ & 1 & 1.019 (0.620-1.675) & 0.94 \\
\hline$\beta$ & & 버 & $1.079(0.960-1.213)$ & 0.202 \\
\hline $\mathrm{FHC}$ & rit & & $0.890(0.778-1.019)$ & 0.091 \\
\hline
\end{tabular}

Figure 2 Forest plot of logistic regression analysis for factors predicting the outcome of 3 weeks of Pavlik harness treatment in DDH children. DDH, developmental dysplasia of the hip.

Table 2 Comparison of ultrasound features during the Pavlik harness treatment between the success group and the failure group

\begin{tabular}{|c|c|c|c|c|c|c|c|c|c|c|c|}
\hline Variable & Group & $\begin{array}{c}\text { Pre- } \\
\text { treatment }\end{array}$ & $1^{\text {st }}$ week & $2^{\text {nd }}$ week & $3^{\text {rd }}$ week & $F_{\text {within group }}$ & $P_{\text {within group }}$ & Fbetween group & $P_{\text {between group }}$ & Finteraction & Pinteraction \\
\hline \multirow[t]{2}{*}{$\alpha$ angle $\left(^{\circ}\right)$} & $\begin{array}{l}\text { Success } \\
\text { group }\end{array}$ & $42.27 \pm 4.43$ & $43.32 \pm 4.60$ & $45.03 \pm 4.77$ & $48.01 \pm 4.91$ & 58.632 & $<0.001$ & 5.071 & 0.025 & 2.901 & 0.036 \\
\hline & $\begin{array}{l}\text { Failure } \\
\text { group }\end{array}$ & $40.19 \pm 4.29$ & $40.64 \pm 4.25$ & $41.04 \pm 4.57$ & $41.39 \pm 4.42$ & & & & & & \\
\hline$\beta$ angle $\left({ }^{\circ}\right)$ & $\begin{array}{l}\text { Failure } \\
\text { group }\end{array}$ & - & $81.68 \pm 4.09$ & $80.57 \pm 4.47$ & $79.79 \pm 4.40$ & & & & & & \\
\hline FHC (\%) & $\begin{array}{l}\text { Success } \\
\text { group }\end{array}$ & $33.64 \pm 8.86$ & $37.56 \pm 9.08$ & $39.33 \pm 9.23$ & $41.17 \pm 9.42$ & 46.937 & $<0.001$ & 2.883 & 0.037 & 2.975 & 0.033 \\
\hline
\end{tabular}

FHC, femoral head coverage; the $\beta$ value of Graf type III was not measured.

could accurately predict the outcome at the first week (AUC $=0.931$, sensitivity $=82.14 \%$, specificity $=97.86 \%$; Figure 5 ).

\section{Discussion}

The sooner the reduction outcome can be determined in the course of Pavlik harness, the earlier the treatment protocol can be changed, and the higher the success rate of reduction in DDH children. This study analyzed the changes of the ultrasonic features ( $\alpha$ and $\beta$ angle and FHC) during the Pavlik harness treatment, and explored the roles of clinical and ultrasonic indicators in predicting 
A

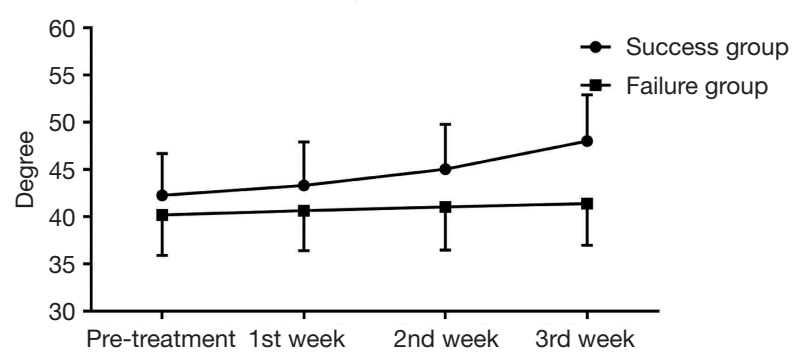

C

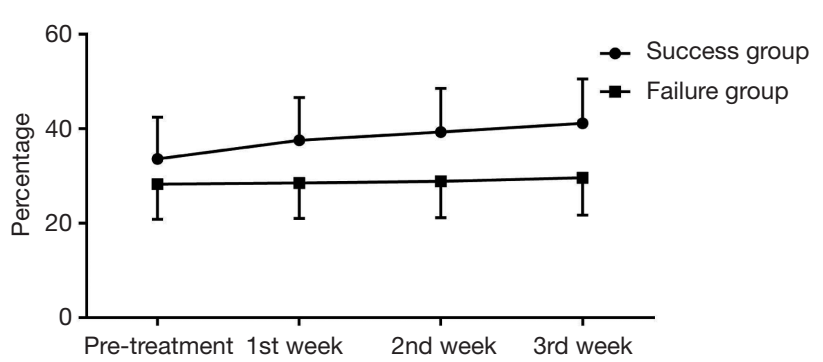

B

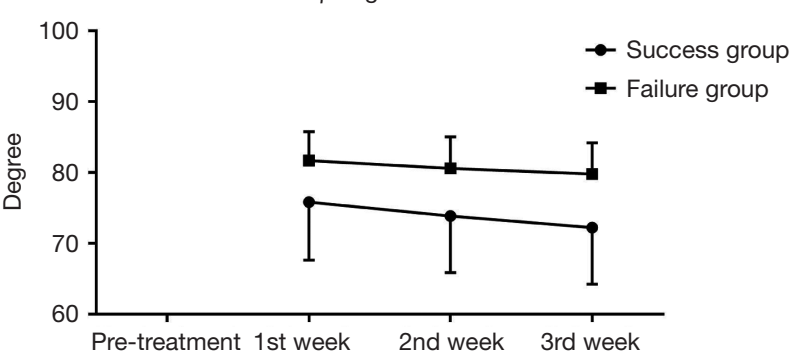

Figure 3 Trends of ultrasound features ( $\alpha$ and $\beta$ and FHC) during the Pavlik harness treatment in DDH children. (A) $\alpha$ angle; (B) $\beta$ angle; (C) FHC. DDH, developmental dysplasia of the hip; FHC, femoral head coverage.

Table 3 ROC analysis of the ultrasound features ( $\alpha$ and $\beta$ angle and FHC) for predicting the outcome of Pavlik harness within 3 weeks

\begin{tabular}{|c|c|c|c|c|c|}
\hline Ultrasound features & AUC & $95 \% \mathrm{Cl}$ & Cutoff point & Sensitivity (\%) & Specificity (\%) \\
\hline$\alpha$ angle $\left({ }^{\circ}\right)$ & 0.590 & $0.521-0.656$ & $45^{\circ}$ & 82.14 & 36.90 \\
\hline$\beta$ angle $\left(^{\circ}\right)$ & 0.766 & $0.703-0.821$ & $79^{\circ}$ & 92.86 & 49.73 \\
\hline $\mathrm{FHC}(\%)$ & 0.689 & $0.623-0.751$ & $29.2 \%$ & 64.29 & 70.59 \\
\hline$\alpha$ angle $\left(^{\circ}\right)$ & 0.639 & $0.571-0.703$ & $39^{\circ}$ & 57.14 & 64.17 \\
\hline$\beta$ angle $\left(^{\circ}\right)$ & 0.838 & $0.782-0.885$ & $79^{\circ}$ & 78.57 & 74.33 \\
\hline $\mathrm{FHC}(\%)$ & 0.698 & $0.632-0.759$ & $29 \%$ & 64.29 & 68.98 \\
\hline \multicolumn{6}{|l|}{ 3rd week } \\
\hline $\mathrm{FHC}(\%)$ & 0.715 & $0.628-0.757$ & $28 \%$ & 62.29 & 68.98 \\
\hline
\end{tabular}

AUC, area under curve; $95 \% \mathrm{Cl}, 95 \%$ confidence interval; $\mathrm{FHC}$, femoral head coverage. 
A

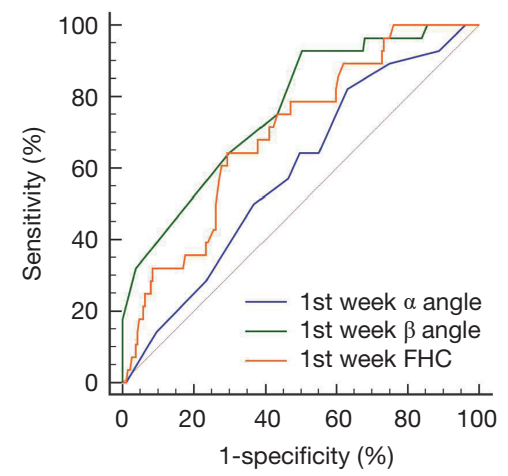

B

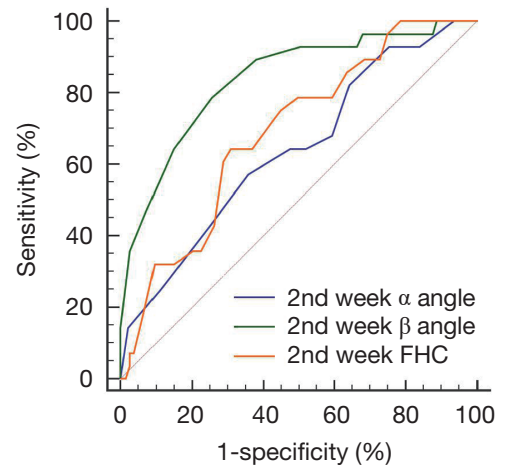

C

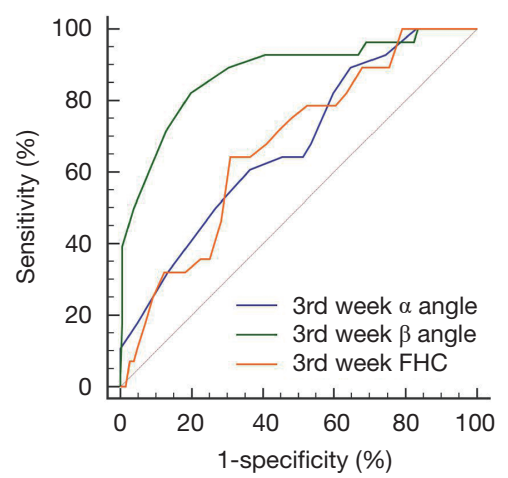

Figure 4 ROC analysis of the ultrasound features ( $\alpha$ and $\beta$ angle and FHC) in predicting the outcome of Pavlik harness within 3 weeks. (A) The first week; (B) the second week; (C) the third week. FHC, femoral head coverage.

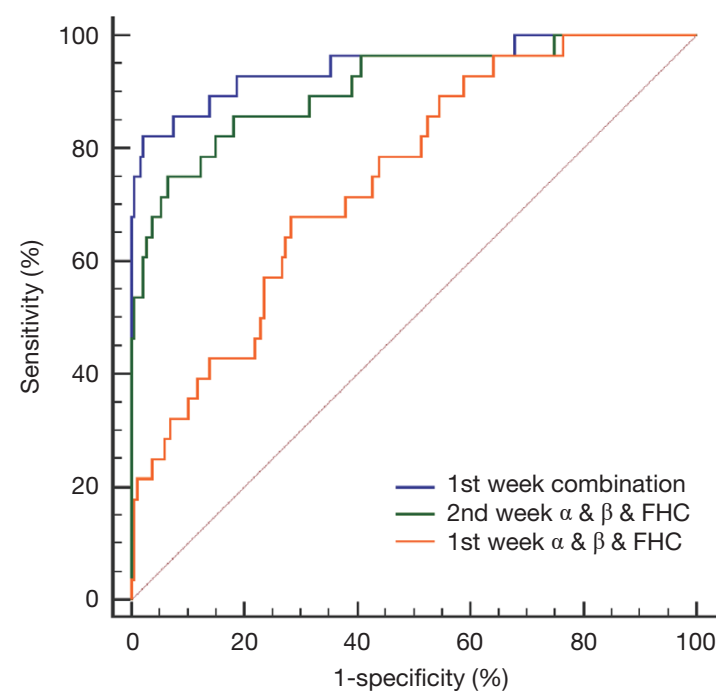

Figure 5 The combined model including the ultrasound features ( $\alpha$ and $\beta$ angle and FHC) and the influencing factors (Graf classification, age at diagnosis, and side of pathology) in predicting the outcome of Pavlik harness treatment after 3 weeks. FHC, femoral head coverage.

the treatment outcome of the Pavlik harness after 3 weeks.

\section{Independent predictors for the 3-week outcome of Pavlik barness treatment}

Graf type III, age at diagnosis, and side of pathology were the independent predictors for the outcome of Pavlik harness in DDH children. Among them, Graf type III had the greatest impact on reduction outcomes likely because children with Graf type III have poor acetabular development in which the acetabular margin cartilage is turned out, and the acetabular cartilage top is pushed up. Hence, reduction is more difficult than in Graf type IIc and type D children (20,21).

The study of Aarvold et al. (22) reported that the success opportunity of Pavlik harness for bilateral hip dislocation was lower than that for the unilateral type, but the difference was not significant. Our study included a larger sample size compared to their study and found that bilateral hip dislocation was a predictor for the reduction outcome that was second only to Graf type III. This is because the treatment protocol for children with bilateral hip dislocation is more difficult. It is necessary to consider the extent of bilateral hip dislocation and adjust the sling angle accordingly. Furthermore, a successful reduction is defined only when bilateral hips are reset, so the incidence of failure is higher than that in DDH children with unilateral dislocation (23).

The age at diagnosis is still controversial as a predictor for reduction outcome. As the age at diagnosis increases, hip dislocation becomes further complicated, and the difficulty of Pavlik Harness treatment becomes greater, thus reducing the success rate (24). However, in a study by Larson et al. (25), for infants younger than 4 weeks, the treatment outcome of Pavlik harness was not significantly related to the age at diagnosis. In addition, with the popularization of ultrasound screening, the difference of age at diagnosis has become less significant. Therefore, though the age at diagnosis in this study was an independent predictor, its effect on reduction outcome was not as significant as Graf classification and side of pathology. 


\section{Changes in ultrasound features during Pavlik harness treatment}

In contrast to clinical indicators, ultrasound features, including $\alpha$ and $\beta$ angle and FHC, can indicate the changes of the hip joint on a week-by-week basis. Salduz et al. (26) reported that $\alpha$ angle was negatively correlated with treatment time, while White et al. (27) found that the increase of $\beta$ angle and the decrease of FHC during treatment were significantly related to the failure of Pavlik harness treatment. The findings from our study support these conclusions. In addition, by comparing the changes in $\alpha$ and $\beta$ angle and FHC before and after treatment between the two groups, we found that the changes of $\alpha$ and $\beta$ angle and FHC in the success group were generally greater than the changes of the failure group. This suggests that in the first 3 weeks of treatment, the faster the changing rate of $\alpha$ and $\beta$ angle and FHC was, the higher the opportunity for successful Pavlik harness treatment would be. In the success group, $\alpha$ angle and FHC were larger while $\beta$ angle was smaller, indicating that monitoring the changes of $\alpha$ and $\beta$ angle and FHC every week can help to evaluate the treatment efficacy.

\section{Accuracy of ultrasonic features in predicting the reduction outcome after 3 weeks of Pavlik harness treatment}

The ROC analysis in this study found that with the advancement of treatment, the accuracy of $\alpha$ and $\beta$ angle and FHC in predicting the outcome gradually improved, and all reached the highest AUC at third week. The accuracy of $\beta$ angle was higher than that of $\alpha$ angle and FHC (but the AUC was still <0.9). This indicates that $\alpha$ angle, $\beta$ angle, or FHC alone cannot accurately predict the reduction outcome after 3 weeks of Pavlik harness treatment.

Therefore, we established prediction models by combining all of the above-mentioned ultrasound features. The accuracy of the combination of the first week of $\alpha$ and $\beta$ angle and FHC for predicting the outcome was still low (AUC =0.746). However, when combined with the second week of features, the prediction accuracy was higher (AUC $>0.9)$, meaning that the combined ultrasound features $(\alpha$ and $\beta$ angle and FHC) at the second week of Pavlik harness treatment could accurately predict the reduction outcome.

In order to explore the opportunity of accurately predicting the treatment efficacy earlier, we combined the first week of ultrasound features with the independent predictors (Graf classification, age at diagnosis, and side of pathology). The results showed that the accuracy of the combination was significantly improved (AUC $=0.931$ ). The advantage of the combined prediction model is that it is expected to predict the reduction outcome of Pavlik harness treatment after 3 weeks in advance, so as to adjust the subsequent treatment plan as soon as possible, which can increase the success rate of other treatments (mostly traction plaster) and reduce the risk of femoral head necrosis.

\section{Limitations and perspective}

Some limitations to this study should be addressed. First, when predicting the eventual efficacy of DDH children, in addition to considering the timing of changing, the impact of changed treatment protocol on hip joint development must also be considered. Moreover, the eventual efficacy will still be affected by the failure of the Pavlik harness even if it has been replaced after 3 weeks. In future research, we plan to explore a method to accurately predict the eventual reduction outcome of DDH children before treatment. For children at risk of poor reduction, treatment with traction plaster may be preferable as it can avoid the adverse effects of treatment change.

\section{Conclusions}

The combined model of ultrasound features ( $\alpha$ and $\beta$ angle and FHC) at the second week of Pavlik harness treatment could accurately predict the reduction outcome after 3 weeks. A combined model consisting of these ultrasound features together with independent predictors (Graf classification, age at diagnosis, and side of pathology) could accurately predict the reduction outcome at the first week. This combined prediction model may help assess the efficacy of Pavlik harness treatment as early as possible, thus facilitating the timely determination of the subsequent treatment protocol and improving the prognosis of DDH children.

\section{Acknowledgments}

Funding: None.

\section{Footnote}

Reporting Checklist: The authors have completed the STARD 
reporting checklist. Available at http://dx.doi.org/10.21037/ apm-20-889

Data Sharing Statement: Available at http://dx.doi. org/10.21037/apm-20-889

Conflicts of Interest: All authors have completed the ICMJE uniform disclosure form (available at http://dx.doi. org/10.21037/apm-20-889). The authors have no conflicts of interest to declare.

Ethical Statement: The authors are accountable for all aspects of the work in ensuring that questions related to the accuracy or integrity of any part of the work are appropriately investigated and resolved. All children's families signed informed consent, and this study was approved by the ethics committee of Ningbo Women \& Children's Hospital (approval ID: NWCH20160217).

Open Access Statement: This is an Open Access article distributed in accordance with the Creative Commons Attribution-NonCommercial-NoDerivs 4.0 International License (CC BY-NC-ND 4.0), which permits the noncommercial replication and distribution of the article with the strict proviso that no changes or edits are made and the original work is properly cited (including links to both the formal publication through the relevant DOI and the license). See: https://creativecommons.org/licenses/by-nc-nd/4.0/.

\section{References}

1. Noordin S, Umer M, Hafeez K, et al. Developmental dysplasia of the hip. Orthop Rev (Pavia) 2010;2:e19.

2. Shaw BA, Segal LS. Evaluation and Referral for Developmental Dysplasia of the Hip in Infants. Pediatrics 2016;138:e20163107.

3. Wassef AJ, Khlopas A, Sodhi N, et al. Use of an offset head center acetabular shell in difficult primary total hip arthroplasties. Ann Transl Med 2019;7:75.

4. Schams M, Labruyere R, Zuse A, et al. Diagnosing developmental dysplasia of the hip using the Graf ultrasound method: risk and protective factor analysis in 11,820 universally screened newborns. Eur J Pediatr 2017;176:1193-200.

5. Paton RW. Screening in Developmental Dysplasia of the Hip (DDH). Surgeon 2017;15:290-6.

6. Pollet V, Percy V, Prior HJ. Relative Risk and Incidence for Developmental Dysplasia of the Hip. J Pediatr
2017;181:202-7.

7. Cooper AP, Doddabasappa SN, Mulpuri K. Evidencebased management of developmental dysplasia of the hip. Orthop Clin North Am 2014;45:341-54.

8. Gornitzky AL, Schaeffer EK, Price CT, et al. Pavlik Harness Disease Revisited: Does Prolonged Treatment of a Dislocated Hip in a Harness Adversely Affect the alpha Angle?. J Pediatr Orthop 2018;38:297-304.

9. Swarup I, Penny CL, Dodwell ER. Developmental dysplasia of the hip: an update on diagnosis and management from birth to 6 months. Curr Opin Pediatr 2018;30:84-92.

10. Ömeroğlu H, Kose N, Akceylan A. Success of Pavlik Harness Treatment Decreases in Patients $>/=4$ Months and in Ultrasonographically Dislocated Hips in Developmental Dysplasia of the Hip. Clin Orthop Relat Res 2016;474:1146-52.

11. Novais EN, Kestel LA, Carry PM, et al. Higher Pavlik Harness Treatment Failure Is Seen in Graf Type IV Ortolani-positive Hips in Males. Clin Orthop Relat Res 2016;474:1847-54.

12. Vaquero-Picado A, Gonzalez-Moran G, Garay EG, et al. Developmental dysplasia of the hip: update of management. EFORT Open Rev 2019;4:548-56.

13. Ibrahim DA, Skaggs DL, Choi PD. Abduction bracing after Pavlik harness failure: an effective alternative to closed reduction and spica casting? J Pediatr Orthop 2013;33:536-9.

14. Tiruveedhula M, Reading IC, Clarke NM. Failed Pavlik harness treatment for DDH as a risk factor for avascular necrosis. J Pediatr Orthop 2015;35:140-3.

15. Zajonz D, Strobel S, Wojan M, et al. (Pavlik harness for the treatment of congenital hip dysplasia types D III and IV). Der Orthopade 2016;45:72-80.

16. Tsukagoshi Y, Kamada H, Takeuchi R, et al. Threedimensional MRI analyses of prereduced femoral head sphericity in patients with developmental dysplasia of the hip after Pavlik harness failure. J Pediatr Orthop B 2018;27:394-8.

17. Graf $R$. The diagnosis of congenital hip-joint dislocation by the ultrasonic Combound treatment. Arch Orthop Trauma Surg 1980;97:117-33.

18. Morin C, Harcke HT, MacEwen GD. The infant hip: realtime US assessment of acetabular development. Radiology 1985;157:673-7.

19. Harcke HT, Clarke NM, Lee MS, et al. Examination of the infant hip with real-time ultrasonography. J Ultrasound Med 1984;3:131-7. 
20. Ömeroglu H. Treatment of developmental dysplasia of the hip with the Pavlik harness in children under six months of age: indications, results and failures. J Child Orthop 2018;12:308-16.

21. Choudry Q, Paton RW. Pavlik harness treatment for pathological developmental dysplasia of the hip: meeting the standard?. J Pediatr Orthop B 2017;26:293-7.

22. Aarvold A, Schaeffer EK, Kelley S, et al. Management of Irreducible Hip Dislocations in Infants With Developmental Dysplasia of the Hip Diagnosed Below 6 Months of Age. J Pediatr Orthop 2019;39:e39-e43.

23. Palocaren T, Rogers K, Haumont T, et al. High failure rate of the Pavlik harness in dislocated hips: is it bilaterality? J Pediatr Orthop 2013;33:530-5.

Cite this article as: $\mathrm{Xu}$ GW, Yang YC, Xu ZH, Liu YL. Ultrasound features predicting the 3-week outcome of Pavlik harness treatment for developmental hip dysplasia. Ann Palliat Med 2020;9(3):1020-1029. doi: 10.21037/apm-20-889
24. Kotlarsky P, Haber R, Bialik V, et al. Developmental dysplasia of the hip: What has changed in the last 20 years? World J Orthop 2015;6:886-901.

25. Larson JE, Patel AR, Weatherford B, et al. Timing of Pavlik Harness Initiation: Can We Wait? J Pediatr Orthop 2019;39:335-8.

26. Salduz A, Demirel M, Akgul T, et al. An analysis of variables affecting the duration of Pavlik harness treatment: Is it possible to predict the duration of treatment? Prosthet Orthot Int 2018;42:299-303.

27. White KK, Sucato DJ, Agrawal S, et al. Ultrasonographic findings in hips with a positive Ortolani sign and their relationship to Pavlik harness failure. J Bone Joint Surg Am 2010;92:113-20. 